\title{
Probing ultrafast energy transfer between excitons and plasmons in the ultrastrong coupling regime
}

Sinan Balci, Coskun Kocabas, Betül Küçüköz, Ahmet Karatay, Elif Akhüseyin, H. Gul Yaglioglu, and Ayhan Elmali

Citation: Appl. Phys. Lett. 105, 051105 (2014);

View online: https://doi.org/10.1063/1.4892360

View Table of Contents: http://aip.scitation.org/toc/apl/105/5

Published by the American Institute of Physics

\section{Articles you may be interested in}

Core-shell gold J-aggregate nanoparticles for highly efficient strong coupling applications

Applied Physics Letters 96, 253107 (2010); 10.1063/1.3456523

Couple molecular excitons to surface plasmon polaritons in an organic-dye-doped nanostructured cavity Applied Physics Letters 108, 193111 (2016); 10.1063/1.4949562

Plasmon-coupled resonance energy transfer: A real-time electrodynamics approach

The Journal of Chemical Physics 146, 064109 (2017); 10.1063/1.4975815

Ultrafast coherent energy transfer with high efficiency based on plasmonic nanostructures

The Journal of Chemical Physics 146, 144101 (2017); 10.1063/1.4979671

Controlling phase of microwaves with active graphene surfaces

Applied Physics Letters 110, 161102 (2017); 10.1063/1.4980087

Charge transfer plasmons: Recent theoretical and experimental developments

Applied Physics Reviews 4, 021104 (2017); 10.1063/1.4982890

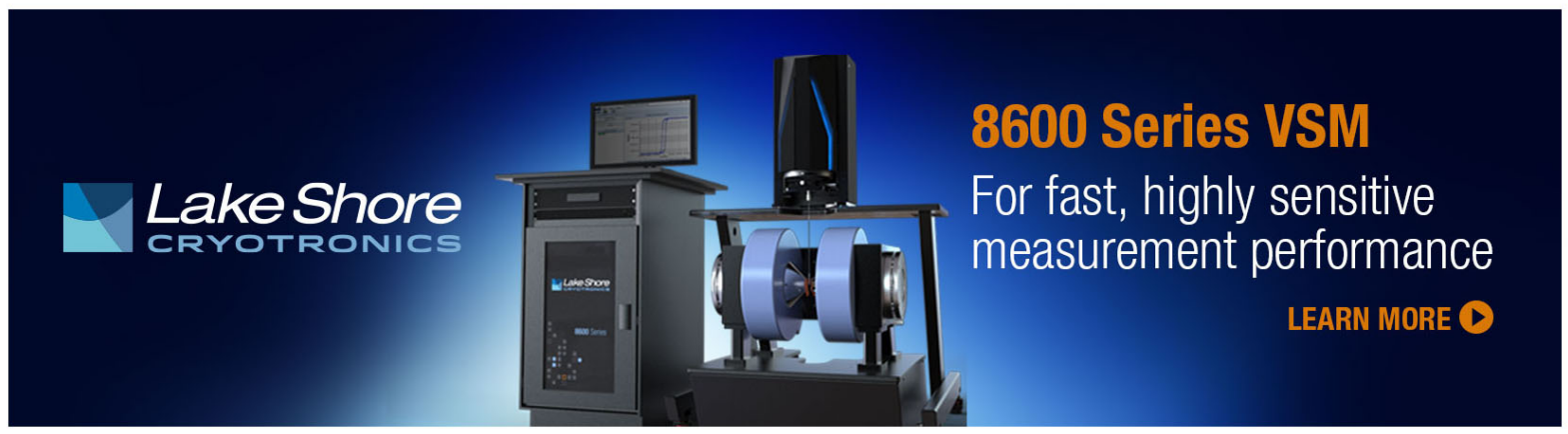




\title{
Probing ultrafast energy transfer between excitons and plasmons in the ultrastrong coupling regime
}

\author{
Sinan Balci, ${ }^{1, a)}$ Coskun Kocabas, ${ }^{2}$ Betül Küçüköz, ${ }^{3}$ Ahmet Karatay, ${ }^{3}$ Elif Akhüseyin, ${ }^{3}$ \\ H. Gul Yaglioglu, ${ }^{3, a)}$ and Ayhan Elmali ${ }^{3}$ \\ ${ }^{1}$ Department of Astronautical Engineering, University of Turkish Aeronautical Association, 06790 Ankara, \\ Turkey \\ ${ }^{2}$ Department of Physics, Bilkent University, 06800 Ankara, Turkey \\ ${ }^{3}$ Department of Engineering Physics, Faculty of Engineering, Ankara University, Besevler, Ankara 06100, \\ Turkey
}

(Received 18 June 2014; accepted 22 July 2014; published online 4 August 2014)

\begin{abstract}
We investigate ultrafast energy transfer between excitons and plasmons in ensembles of core-shell type nanoparticles consisting of metal core covered with a concentric thin J-aggregate (JA) shell. The high electric field localization by the Ag nanoprisms and the high oscillator strength of the JAs allow us to probe this interaction in the ultrastrong plasmon-exciton coupling regime. Linear and nonlinear optical properties of the coupled system have been measured using transient absorption spectroscopy revealing that the hybrid system shows half-plasmonic and half-excitonic properties. The tunability of the nanoprism plasmon resonance provides a flexible platform to study the dynamics of the hybrid state in a broad range of wavelengths. (C) 2014 AIP Publishing LLC.

[http://dx.doi.org/10.1063/1.4892360]
\end{abstract}

The control of light-matter interaction at the nanoscale dimension has attracted a considerable interest in recent years. This is due to the fundamental interest, e.g., spectroscopy and nonlinear optics, and the practical interest, e.g., light emitting diodes and solar cells. ${ }^{1-6}$ The interest has been mainly fueled by the recent advancements achieved in nanoscale fabrication and chemical synthesis of metallic nanostructures-supporting surface plasmon polaritons (SPPs)_and semiconductor nanostructures_-supporting excitons - with a very high degree of structural precision and accuracy. SPPs guide and manipulate light on the nanoscale. On the other hand, electronic excitation in semiconductors generates excitons. The hybrid metal-semiconductor nanostructure is formed when metallic nanostructures are carefully brought into physical contact in an appropriate condition with the semiconductor nanostructures leading to appealing, tunable optical properties. Up until now, coupling between SPPs and excitons extensively studied by many groups has resulted in light-matter coupling regimes ranging all the way from weak-coupling to ultrastrong-coupling. ${ }^{7-11}$ In the weak-coupling regime where damping rates of plasmon and exciton prevail over plasmon-exciton coupling parameter, the wavefunction of the excitonic system is slightly modified or not altered resulting in modification of absorption or emission properties of the semiconductors. Therefore, in this regime, no new optical modes are generated. However, in the strong coupling regime where the plasmon-exciton coupling parameter now prevails over damping rates results in formation of polariton states. Consequently, these new states show two polariton branches called a lower polariton branch (LPB) and an upper polariton branch (UPB). The branches are separated by an energy value called Rabi splitting energy, which is an indicator of the plasmon-exciton interaction strength. It is defined as $\hbar \Omega_{R}=\left(4 V^{2}-\left(\gamma_{s p p}-\gamma_{x}\right)^{2}\right)^{112}$, where $\hbar \Omega_{R}$

${ }^{\text {a)} E l e c t r o n i c ~ a d d r e s s e s: ~ s b a l c i @ t h k . e d u . t r ~ a n d ~ g u l . y a g l i o g l u @ e n g . a n k a r a . e d u . t r ~}$ is the Rabi splitting energy, $V$ is the coupling parameter, $\gamma_{s p p}$ is the line width of the SPPs, and $\gamma_{x}$ is the linewidth of the excitons. Strong coupling has been observed, for example, in optical microcavities, ${ }^{1}$ in a variety of metal nanostructures, and metal thin films in various configurations. In this regime, the hybrid structures show half plasmon-like properties and half exciton-like properties. Large oscillator strength of organic semiconductors and large electric field localizations of plasmonic nanostructures are the main reasons for the observation of strong coupling. Surprisingly, when the Rabi splitting energy value is greater than $\sim 15 \%$ of the molecular transition energy, a completely new regime called ultrastrong-coupling regime is reached. ${ }^{7}$

Recently, ultrastrong coupling has been observed in a hybrid nanostructure of J-aggregates (JAs) self-assembled on metal nanoprisms, ${ }^{9}$ and also in single nanoparticle level. ${ }^{10}$ In the ultrastrong light-matter interaction regime, it is possible to greatly modify and tune the optical properties of the matter. For example, the absorbed energy can be exchanged reversibly and coherently between the plasmonic and excitonic modes in the excited state many times before the coupled hybrid system decays back to its ground state. ${ }^{11}$ Therefore, a lot of efforts have been recently devoted to understand energy transfer mechanism in this regime. The dynamics of the energy exchange has been extensively studied in a variety of configurations. ${ }^{12-16}$ For example, exciton-photon coupling dynamics has been studied in optical microcavities. ${ }^{16}$ The hybrid nanostructures of Au-nanoshell-JA have been used to study the dynamical behavior of the coupled system. ${ }^{15}$ The coupling between the excitons of CdSe nanocrystals and SPPs has been studied by transient pump-probe measurements. ${ }^{17,18}$

In this Letter, we report on the ultrafast energy transfer in metal-organic hybrid nanostructures [Fig. 1]. The system provides a unique configuration to probe the ultrastrong coupling regime because the hybrid system does not contain any 


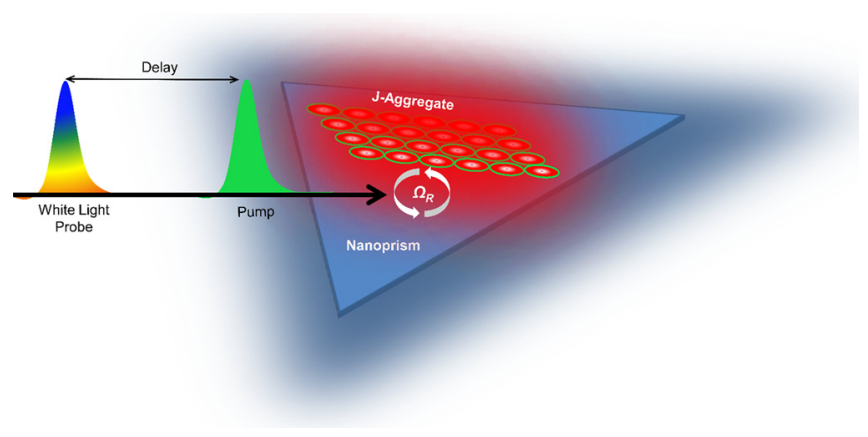

FIG. 1. Schematic representation of the energy transfer occurring between the excitons of JAs and localized plasmons of Ag nanoprisms. Individual TDBC molecules self-assemble on the surface of the Ag nanoprisms and hence construct JA nanostructures. The dynamics of the energy exchange has been studied by using transient absorption spectroscopy.

uncoupled Ag nanoprisms or JAs. We extensively study the energy transfer dynamics in the ultrastrong coupling regime. Hybrid system has the following properties: (1) The plasmon resonance of the nanoprism can be easily tunable. (2) JAs selectively self-assemble on the Ag nanoprisms and free dye molecules are removed by centrifugation. Most of the previously studied plexitonic systems concurrently contain bare and coupled molecules in the medium making the energy transfer mechanisms particularly difficult to interpret. (3) The hybrid nanostructure exhibits very large Rabi splitting energies because of the high oscillator strength of JAs and very high electric field localization emanating from the sharp corners of the metal nanoprism.

The Ag nanoprisms have been wet chemically synthesized. ${ }^{9,19-22}$ We used a cyanine dye 5, 5', 6, 6'-tetrachlorodi-(4-sulfobutyl) benzimidazolocarbocyanine (TDBC) forming JAs at high concentration as the exciton source. The hybrid nanostructure of the $\mathrm{Ag}$ nanoprism-JA is formed by selfassembly of the dye molecules on the Ag nanoprism surfaces and thus conformal coating of the nanoprisms can be achieved. $^{9,23-25}$ This simple synthesis of Ag nanoprism-JAs hybrid nanostructure is highly reproducible allowing preparation of high quality hybrid nanostructures for plasmonic applications. ${ }^{9}$
Transient absorption measurements were carried out with femtosecond temporal resolution using a femtosecond pump-probe method [Fig. 1] utilizing Ti:sapphire laser amplifier-optical parametric amplifier system (Spectra Physics) with 44 fs pulse duration and $1 \mathrm{kHz}$ repetition rate. Commercial pump-probe experimental setup with whitelight continuum probe beam (Spectra Physics) was used. Experiments were performed in transmission geometry and at $0.25 \mu \mathrm{J} / \mathrm{cm}^{2}$ pump fluencies. Pulse duration is $100 \mathrm{fs}$. According to our sign convention, a positive sign of pumpprobe data $(\Delta \mathrm{T} / \mathrm{T})$ corresponds to a decrease of transmission (excited state absorption (ESA)), whereas a negative sign of $\Delta \mathrm{T} / \mathrm{T}$ corresponds to an increase of transmission (bleach signal).

We measured the ground state extinction spectra of the Ag nanoprisms [Fig. 2(a)], the JAs [Fig. 2(b)], and the hybrid nanostructures [Fig. 2(c)]. Similarly, extinction spectra from the individual silver nanorods coated with dye molecules have been previously shown. ${ }^{26}$ After self-assembly of the dye molecules on the surface of the Ag nanoprisms, ${ }^{9}$ the color of the hybrid system turns into purple [Fig. 2(c)] due to quenching of the plasmon resonance peak-a dip in the spectrum-located at the exciton resonance energy level. ${ }^{27}$

Then, we measured the transient absorption spectra $(\Delta \mathrm{T} / \mathrm{T})$ of the Ag nanoprism [Fig. 2(d)], the JA [Fig. 2(e)], and the hybrid nanostructures [Fig. 2(f)]. The spectra of the Ag nanoprisms show a typical absorption decrease at highenergy side of the in-plane dipole resonance band but absorption increase at the low energy side. Previously, the same feature has been observed in the Ag nanoprisms and attributed to a red-sifting of the dipole resonance band. ${ }^{28}$ The spectra of the JAs also show typical features as previously observed. ${ }^{29}$ The positive absorption peak corresponds to the excited state absorption from one-exciton to twoexciton states; however, negative absorption signal corresponds to the ground state bleaching and stimulated emission. In a similar way, representative transient absorption spectra of nanoprism-JA are shown in Fig. 2(f) demonstrating completely different features from the spectra of the nanoprism and the JAs. It is clear that as in the case of the
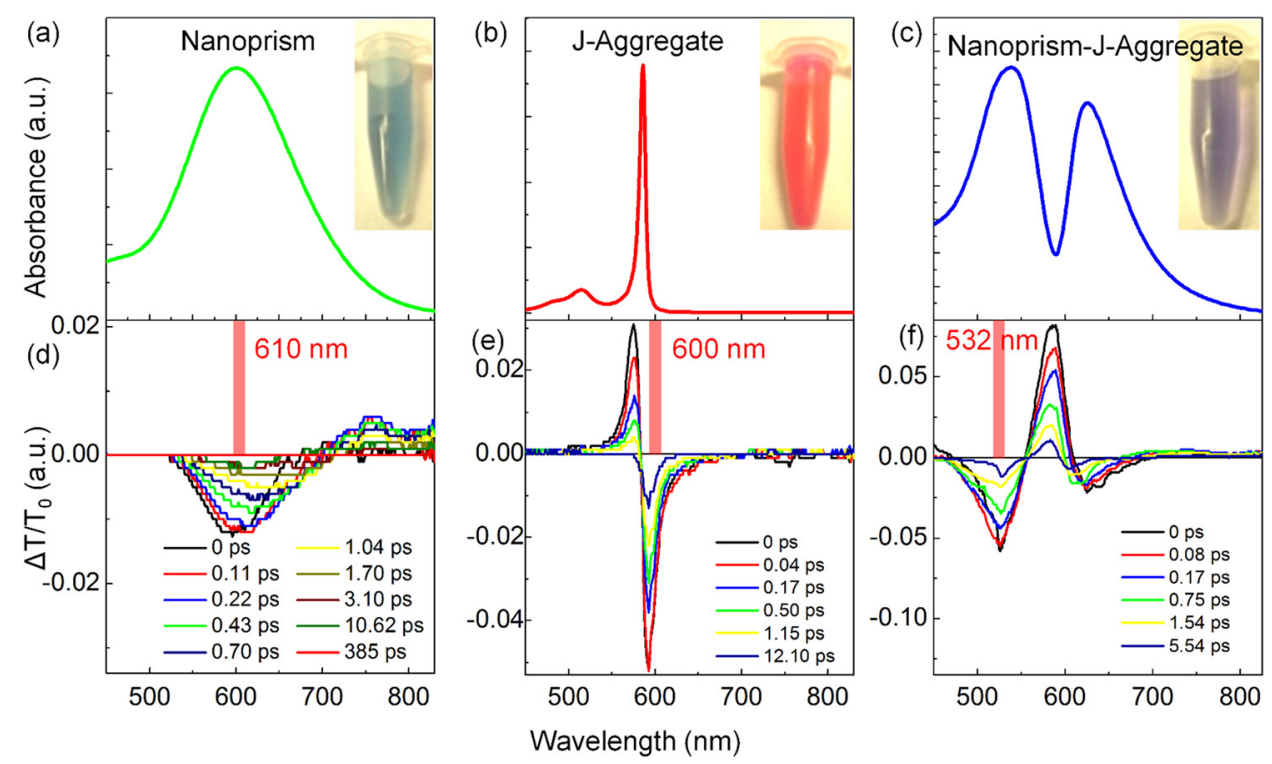

FIG. 2. Linear absorption spectra of (a) Ag nanoprism, (b) JA, and (c) $\mathrm{Ag}$ nanoprism-JA hybrid nanostructure. The insets show the photographs of the samples. Transient absorption spectra of (d) Ag nanoprism, (e) JA, and (f) Ag nanoprism-JA hybrid nanostructure acquired at zero time delay. The time delays are measured in units of picoseconds (ps). The vertical red lines correspond to central wavelength of the pump pulses. 

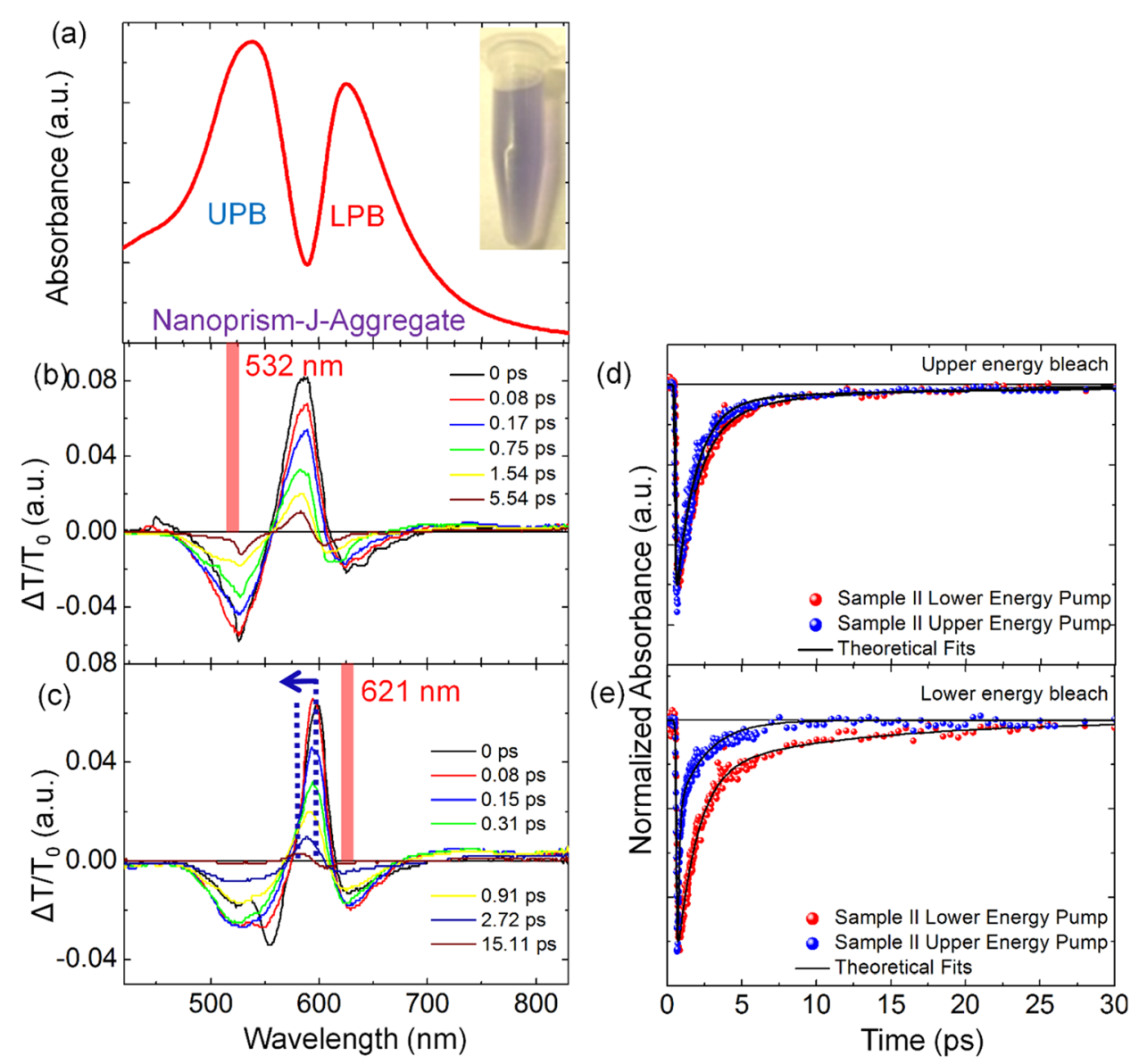

FIG. 3. (a) Linear absorption spectrum of the hybrid nanostructure. (b) UPB is selectively excited at $532 \mathrm{~nm}$. Transient absorption spectra of the sample for varying time delays. (c) LPB is selectively excited at $621 \mathrm{~nm}$. Transient absorption spectra of the sample for varying time delays. The blue arrow indicates the direction of the wavelength shift of the upper energy bleach signal observed upon increase in the time delays. (d) Decay curves of the upper energy bleach signal pumped in the UPB and LPB for sample II. (e) Decay curves of the upper bleach signal pumped in the UPB and LPB for sample II. The lower polaritons are short lived. The vertical red lines correspond to central wavelength of the pump pulses.

ground state absorption spectrum shown in Fig. 2(c), the plasmon band has been quenched at the position of the exciton resonance energy value.

To understand the effect of the coupling strength on the dynamics of ultrafast energy transfer, we have prepared several nanoprism-JAs samples yielding plasmon resonance between 1.75 and $2.75 \mathrm{eV}$. While Fig. 3(a) shows the linear absorption spectrum, Figs. 3(b) and 3(c) show representative transient absorption spectra for various time delays pumped in the UPB at $532 \mathrm{~nm}$ and in the LPB at $621 \mathrm{~nm}$, respectively. The pump signal promotes electrons/holes to the high energy states, and time-delayed probe signal measures how the transmission varies as a function of the delay between the pump and probe signals. Transmission pump-probe spectra show bleach signals (negative absorption) at around $525 \mathrm{~nm}$ and $625 \mathrm{~nm}$ wavelengths along with a positive signal between two bleach signals regardless of the pumping wavelengths. Observed bleach signals at 525 and $625 \mathrm{~nm}$ wavelengths originate from the hybrid states observed in the linear absorption spectrum [Figs. 2(c) and 3(a)]. In a previous study, pump-probe measurements on similar coupled systems show only positive signal and bleach signal on the lower energy side of the positive signal. ${ }^{30}$ The fact that both bleach signals appearing simultaneously in the pump-probe data is due to the ultrastrong coupling and the lack of the bare JAs and nanoprisms in our samples. Bleach signals appearing at zero pump and probe time delays for both UPB and LPB are the indication of the coherent energy exchange between excitons and plasmons. Previously, the positive signal is assigned to ESA. ${ }^{15,30}$ The clear ESA signal among bleach signals is composed of two peaks varying in magnitude with the time delay as it has also been recently observed. ${ }^{30}$ The fast and the slow components of this signal can be attributed to ESA from plasmons and also ESA from one-exciton to two-exciton state transitions, respectively. ${ }^{15}$ The other possibility for the positive signal is the temporary electron transfer from the dye molecules to the metal nanoparticles. In this sense, the coupling strength decreases because of the decrease in the number of excitons, leading to evolution of the center positive signal. However, according to previous works, this process is faster than $50 \mathrm{fs}^{18}$ The time resolution of our experimental setup is $100 \mathrm{fs}$. Therefore, we may not be able to see electron transfer contribution to the positive signal. Surprisingly, another bleach signal appears at $550 \mathrm{~nm}$ within 100 fs only for LPB [Fig. 3(c)]. Similar mixed, complex transient bleach signal appearing at earlier times has been recently observed for the coupling of JAs excitons and Au nanoshells plasmons. ${ }^{15}$ It has been attributed to the strong modification of the excitonic states due to the strong coupling. ${ }^{15}$ It is also known that strong coupling results in shifting and splitting of exciton energy levels. ${ }^{31}$ Therefore, the observed extra bleach signal indicates that SPPs and excitons interact strongly and coherently in sample II. The lifetimes of the decay curves of the upper energy bleach signals $(525 \mathrm{~nm})$ for UPB and LPB pumping [Fig. 2(d)] are almost the same ( $\sim 1.5 \mathrm{ps})$. However, the lifetime of the decay curves of the lower energy bleach signal $(625 \mathrm{~nm})$ is shorter for UPB ( $\sim 2 \mathrm{ps})$ than LPB $(\sim 12 \mathrm{ps})$. This is due to the nonradiative energy transfer from the UPB to the LPB. ${ }^{31}$ 
(a)
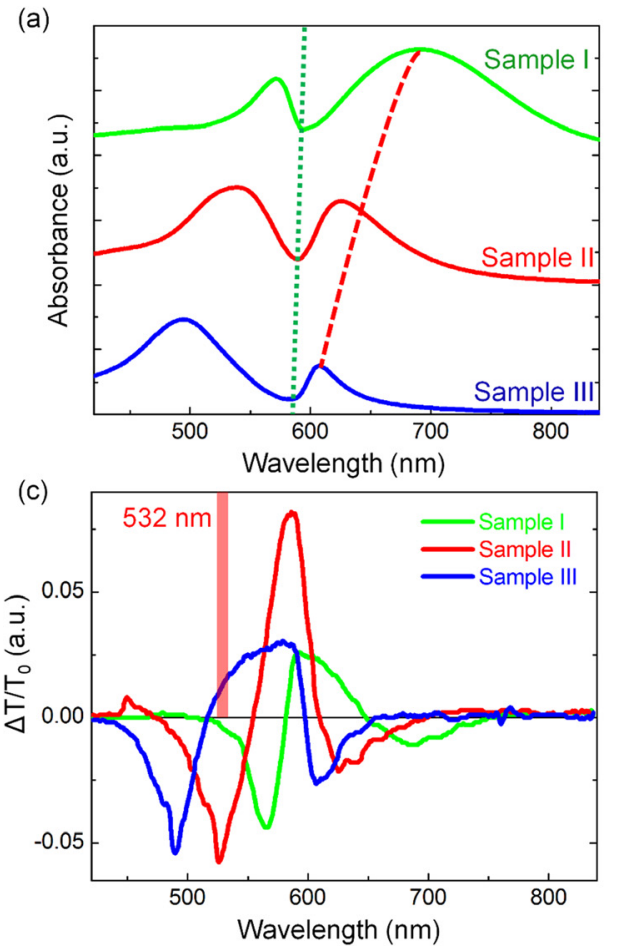

(b)

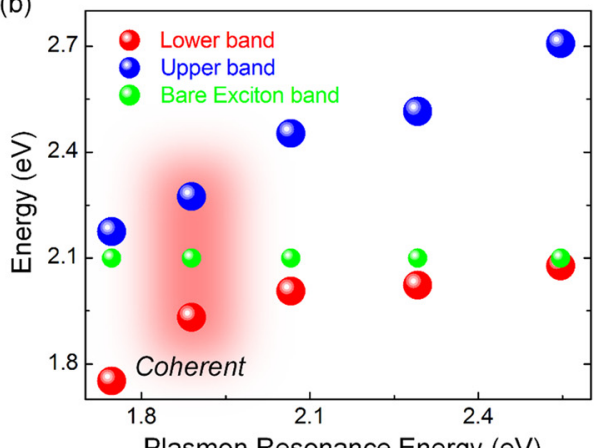

(d)

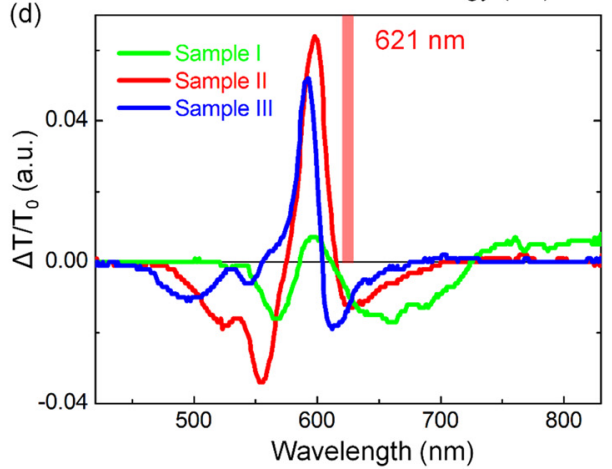

FIG. 4. (a) Absorption spectra of the hybrid nanostructure with varying the nanoprism sizes. (b) Dispersion curve of the hybrid nanostructures. Moving away from the exciton resonance energy in the dispersion curve reveals that the hybrid system becomes more plasmonlike. Transient absorption spectra of the samples I, II, and III at $\mathrm{t}=0$ time delay (c) pumped at $532 \mathrm{~nm}$ and (d) pumped at $621 \mathrm{~nm}$. The vertical red lines correspond to central wavelength of the pump pulses.
In order to understand the energy transfer dynamics as a function of plasmon resonance, we measured the transmission pump-probe spectra of the samples having varied Ag nanoprism sizes [Fig. 4(a)]. As the plasmon mode is tuned closer to the exciton mode, the ultrastrong-coupling is observed [Fig. 4(b)]. When the two modes are on resonance, the upper and lower polariton modes yield equal intensities. Hybrid nanostructure shows coherent polaritonic modes formed along UPB and LPB only for a limited Ag nanoprism size range [Fig. 4(b)]. In fact, the coupling strength depends on the exciton damping and the plasmon damping. ${ }^{31-33}$ The coupling strength reduces for the smaller and larger Ag nanoprism sizes and thus the coherency sharply decreases [Fig. 4(b)]. Figs. 4(c) and 4(d) show the spectra of the UPB and LPB at zero pump and probe time delays, respectively. The spectra show that the observed shift of UPB and LPB to higher energy levels in different samples provides shifting the whole pump-probe spectra to higher energies. However, due to the coherent coupling observed, sample II exhibits larger bleach and transient absorption signals than other samples for both pumping energies. Large energy separation between the two peaks within the ESA signal for sample III allows comparing these peaks for UPB and LPB. Comparison reveals that while faster component of ESA signal at lower energy is larger for LPB, slower component of ESA signal at higher energy gets larger for UPB. In another words, while ESA of plasmons is dominant for LPB, ESA between one-exciton to two-exciton states gets larger for UPB. Since UPB pumping causes larger bleach signal at longer wavelength and increased ESA, double peak of the transient bleach signal at longer wavelength side seen for LPB does not appear for UPB. Splitting of higher energy bleach signal is due to the large coherent coupling. On the other hand, coupling strength also affects the lifetime of the bleach signals. Figure 5 shows the time evolution of the bleach signals. The lifetimes of the upper bleach signal and lower bleach signal become longer for the sample with the stronger coupling strength (sample II). In the strong coupling regime, electron oscillates between dye's excitons and metal's plasmons. Since this process is shorter than the time resolution in our experiments, this process does not affect the decays of the bleach signals. Therefore, sample II has the longest lifetime due to the largest coupling strength. Since sample I has the lowest coupling strength, decaying electron from excited state may not oscillate back and therefore, excited state population may decrease faster than that of sample II. Based on the experimental findings, schematic energy diagram of the hybrid system can be represented with the energy diagram of strongly coupled system that has been observed recently, ${ }^{32}$ Fig. 6.

In summary, we measured linear and nonlinear optical properties of the hybrid nanostructure of JAs and Ag nanoprisms. JAs are selectively self-assembled on Ag nanoprisms. Since our samples do not have any uncoupled plasmonic and excitonic modes, the transient absorption

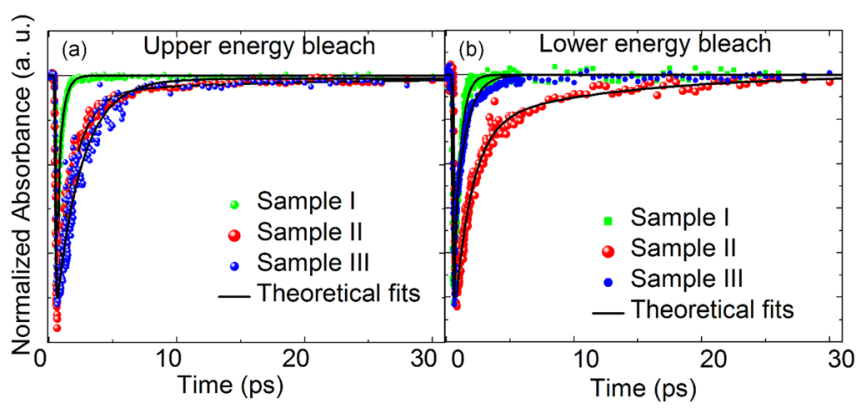

FIG. 5. (a) The time evolution of the upper energy bleach signals pumped in the UPB for samples I, II, and III. (b) The time evolution of the lower energy bleach signals pumped in the LPB for samples I, II, and III. 


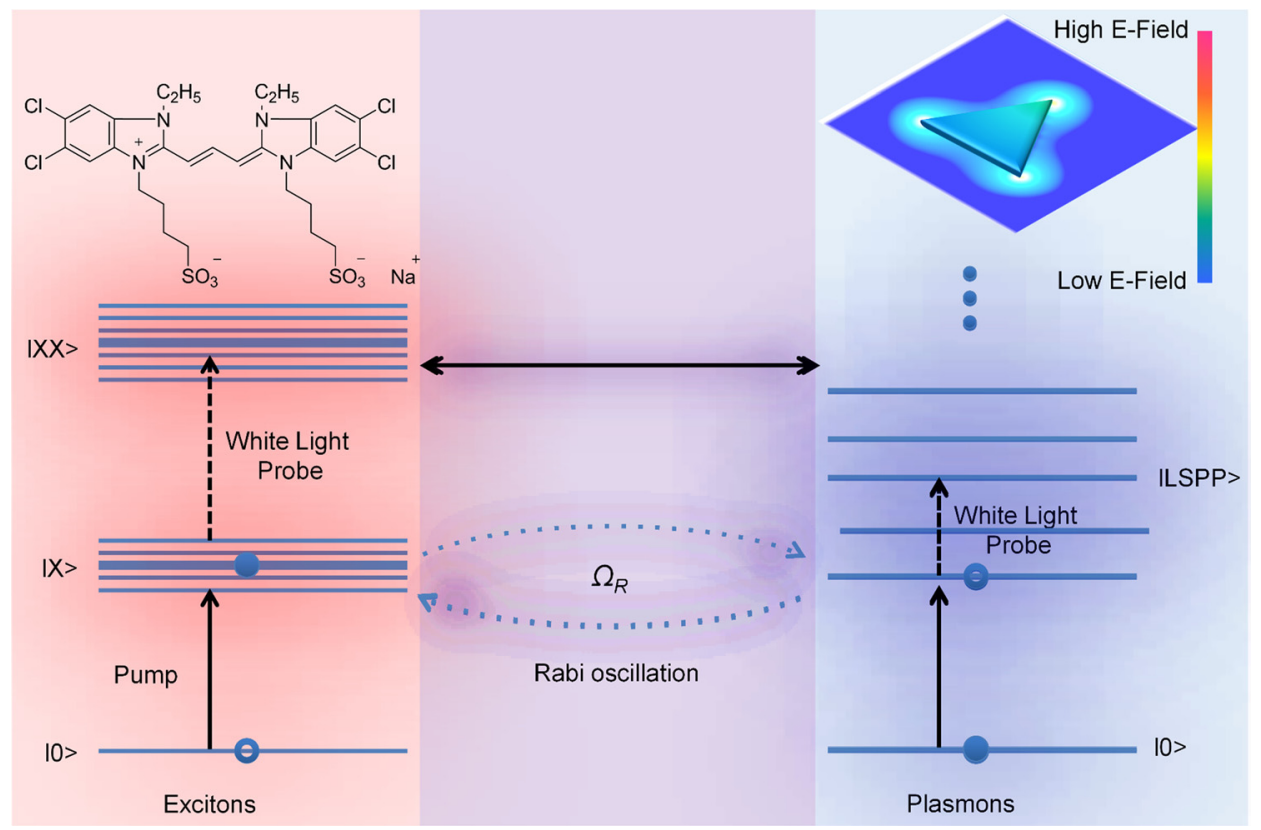

FIG. 6. Schematic representation of the energy diagram and optical transitions in the hybrid nanostructure. IX $>$ and IXX $>$ represent single exciton state and biexciton state, respectively. I0 $>$ and ILSPP $>$ show ground state and plasmonic state, respectively. Dotted and solid vertical arrows represent the optical transitions in excitons and plasmons. The dashed and solid horizontal arrows point the coupling. $\Omega_{R}$ indicates the Rabi oscillation frequency. Electric field localization of a single Ag nanoprism under the resonance condition is calculated by using finite-difference-time-domain method.

experiments reveal dynamics of the energy transfer in the ultrastrong coupling regime. We observed that the lower polaritons are long lived, whereas the upper polaritons are short lived due to the radiationless transitions from the upper polariton branch to the lower polariton branch. Pumping the hybrid sample at the UPB or LPB wavelengths does not make significant differences because the hybrid system shows half-plasmon-like and half-exciton-like properties. Furthermore, the tunability of nanoprism plasmon resonance allows us to study energy transfer dynamics in a broad range of wavelengths. The results would be interesting to understand energy transfer dynamics at the nanoscale dimension and boost the performance of the plasmonic devices and systems.

This work has been supported by a Grant No. 112T091 from TUBITAK.

${ }^{1}$ D. G. Lidzey, D. D. C. Bradley, M. S. Skolnick, T. Virgili, S. Walker, and D. M. Whittaker, Nature 395, 53 (1998).

${ }^{2}$ J. Bellessa, C. Bonnand, J. C. Plenet, and J. Mugnier, Phys. Rev. Lett. 93, 036404 (2004).

${ }^{3}$ D. E. Gomez, K. C. Vernon, P. Mulvaney, and T. J. Davis, Nano Lett. 10, 274 (2010).

${ }^{4}$ G. P. Wiederrecht, G. A. Wurtz, and J. Hranisavljevic, Nano Lett. 4, 2121 (2004).

${ }^{5}$ C. Bonnand, J. Bellessa, and J. C. Plenet, Phys. Rev. B 73, 245330 (2006). ${ }^{6}$ K. Aydin, V. E. Ferry, R. M. Briggs, and H. A. Atwater, Nat. Commun. 2, 517 (2011).

${ }^{7}$ G. Scalari, C. Maissen, D. Turcinkova, D. Hagenmuller, S. De Liberato, C. Ciuti, C. Reichl, D. Schuh, W. Wegscheider, M. Beck, and J. Faist, Science 335, 1323 (2012).

${ }^{8}$ J. A. Hutchison, T. Schwartz, C. Genet, E. Devaux, and T. W. Ebbesen, Angew. Chem., Int. Ed. 51, 1592 (2012).

${ }^{9}$ S. Balci, Opt. Lett. 38, 4498 (2013).

${ }^{10}$ A. E. Schlather, N. Large, A. S. Urban, P. Nordlander, and N. J. Halas, Nano Lett. 13, 3281 (2013).

${ }^{11}$ F. Nagasawa, M. Takase, and K. Murakoshi, J. Phys. Chem. Lett. 5, 14 (2014).
${ }^{12}$ J. Hranisavljevic, N. M. Dimitrijevic, G. A. Wurtz, and G. P. Wiederrecht, J. Am. Chem. Soc. 124, 4536 (2002).

${ }^{13}$ M. Sukharev, T. Seideman, R. J. Gordon, A. Salomon, and Y. Prior, ACS Nano 8, 807 (2014).

${ }^{14}$ D. M. Coles, P. Michetti, C. Clark, W. C. Tsoi, A. M. Adawi, J. S. Kim, and D. G. Lidzey, Adv. Funct. Mater. 21, 3691 (2011).

${ }^{15}$ N. T. Fofang, N. K. Grady, Z. Fan, A. O. Govorov, and N. Halas, Nano Lett. 11, 1556 (2011).

${ }^{16}$ T. Schwartz, J. A. Hutchison, J. Leonard, C. Genet, S. Haacke, and T. W. Ebbesen, ChemPhysChem 14, 125 (2013).

${ }^{17}$ D. E. Gomez, S. S. Lo, T. J. Davis, and G. V. Hartland, J. Phys. Chem. B 117, 4340 (2013).

${ }^{18}$ P. Vasa, W. Wang, R. Pomraenke, M. Lammers, M. Maiuri, C. Manzoni, G. Cerullo, and C. Lienau, Nat. Photonics 7, 128 (2013).

${ }^{19}$ L. J. Sherry, R. Jin, C. A. Mirkin, G. C. Schatz, and R. Van Duyne, Nano Lett. 6, 2060 (2006).

${ }^{20}$ J. Goebl, Q. Zhang, L. He, and Y. Yin, Angew. Chem., Int. Ed. 51, 552 (2012).

${ }^{21}$ D. Aherne, D. M. Ledwith, M. Gara, and J. M. Kelly, Adv. Funct. Mater. 18, 2005 (2008).

${ }^{22}$ D. M. Ledwith, A. M. Whelan, and J. M. Kelly, J. Mater. Chem. 17, 2459 (2007).

${ }^{23}$ N. Kometani, M. Tsubonishi, T. Fujita, K. Asami, and Y. Yonezawa, Langmuir 17, 578 (2001).

${ }^{24}$ D. D. Lekeufack, A. Brioude, A. W. Coleman, P. Miele, J. Bellessa, L. De Zeng, and P. Stadelmann, Appl. Phys. Lett. 96, 253107 (2010).

${ }^{25}$ A. Yoshida and N. Kometani, J. Phys. Chem. C 114, 2867 (2010).

${ }^{26}$ G. Zengin, G. Johansson, P. Johansson, T. J. Antosiewicz, M. Kall, and T. Shegai, Sci. Rep. 3, 3074 (2013).

${ }^{27}$ N. T. Fofang, T. H. Park, O. Neumann, N. A. Mirin, P. Nordlander, and N. J. Halas, Nano Lett. 8, 3481 (2008).

${ }^{28}$ N. Okada, Y. Hamanaka, A. Nakamura, I. Pastoriza-Santos, and L. M. Liz-Marzan, J. Phys. Chem. B 108, 8751 (2004).

${ }^{29}$ G. Yaglioglu, R. Dorsinville, and S. Ozcelik, J. Appl. Phys. 94, 3143 (2003).

${ }^{30}$ G. P. Wiederrecht, G. A. Wurtz, and A. Bouhelier, Chem. Phys. Lett. 461, 171 (2008).

${ }^{31}$ M. Ringler, A. Schwemer, M. Wunderlich, A. Nichtl, K. Kürzinger, T. A. Klar, and J. Feldmann, Phys. Rev. Lett. 100, 203002 (2008).

${ }^{32}$ S. Wang, T. Hutchison, C. Genet, and T. W. Ebbesen, J. Phys. Chem. Lett. 5, 1433 (2014).

${ }^{33}$ W. Wang, P. Vasa, R. Pomraenke, R. Vogelgesang, A. D. Sio, E. Sommer, M. Maiuri, C. Manzoni, G. Cerullo, and C. Lienau, ACS Nano 8, 1056 (2014). 